\title{
A Methodology for the Investigation of Speaker's Knowledge of Structure in Athabaskan
}

\author{
JOYCE MCDONOUGH \& RACHEL SUSSMAN \\ University of Rochester
}

\section{Introduction}

The purpose of this paper is to investigate methodologies that get at speakers' tacit knowledge of structure in polysynthetic languages. As linguists we need theoretical structures that both link languages to other languages and are internally cohesive. Polysynthetic languages pose a particular challenge to linguistic theories because of the internal complexity of the word. Polysynthesis generally refers to language types in which the verbal structure is semantically rich, morphologically complex with an opaque morpheme structure. In polysynthetic languages, word formation is often characterized as an often highly abstract concatenation of virtual morphemes that are conditioned by post hoc morphophonemic rules. However in light of their learnability and their overall long-term stability as polysynthetic, a more parsimonious model is likely to have an advantage over abstract models where internal structure is obscure. Adding to the problem, crucially, given that polysynthetic languages as a rule are often severely under-documented, the availability of data on polysynthesis in no way is comparable to that found with better-studied languages like English, which places us at a severe disadvantage in our understanding of them. With pre-theoretic assumptions in place concerning the nature of polysynthesis, the models resulting from research naturally tend toward translations of polysynthesis into analytic frameworks. We consider such approaches to be limited in their productivity and insight; the rest of this paper focuses on the development of new methodologies for the investigation of polysynthetic languages using Navajo, a canonical example of a polysynthetic language as our test case, expandable to the Athabaskan family.

Some of the essential issues presented by polysynthesis are: the nature of lexical productivity, the structure of a verb-based lexicon, the nature of evidence for theory, techniques of language documentation, and the nature of language change in lexically complex languages. 


\section{The Young and Morgan Grammars}

Important to this enterprise is the existence of the Young and Morgan (YM) grammars and dictionaries, in particular 1980 and 1987, which represent extensive work on the documentation of the Navajo language and the patterns that exist in the language family. One of the more striking aspects of this opus is that the dictionaries are word based. Already, this runs counter to previously established concatenative models. If the morphemes are indeed fully productive, we might expect the lexicon to be a list of the more "productive" morphemes and a set of concatenation rules; this is a standard approach. However, as those who work on polysynthetic languages know, the word is the principal level of sound/meaning pairing. YM refer to fully inflected forms as lexical items; it is at this level that meaning is assigned. We take this as a starting point. If individual units/morphemes exist within the word, they will emerge and speakers will exhibit knowledge of them.

Central to the YM opus on Navajo is the word formation system they developed for their dictionaries. They use a paradigm-based system consisting of two basic units, each exhibiting conjugational variation. For the purpose of this paper, we will call them simply the pre-stem and the stem domains, and refer the reader to discussion of their specific structure. The two parts are combined to form a fully inflected lexical word.

The insight that Young and Morgan provide is that, even in this highly polymorphic language family, the word is still the primary unit of lexical access. This fact calls up issues of morphemic productivity and lexicalization. The two principal parts of the verb are roughly comparable to the stem and pre-stem domains and make up the core verb. Every word consists of at least the bases of these two units, which exhibit paradigmatic or conjugational variation. In their dictionaries, YM $(1980,1987)$ have developed an ingenious word formation algorithm that allows the combination of these two paradigm types (Base paradigms and stem paradigms) into a word and associates the word entry to the full set of conjugations and inflections that are possible for that $w o r d .{ }^{1}$ It is in this sense that word formation is a conjoining of the two units. In the following example, the verb word yishcha 'I cry' is broken into its two component parts, a bisyllabic unit (in the dictionary, this word is linked, via the pre-stem, to a set of paradigms that it participates in as a meaning unit). This is an example of a minimal or core verb. We refer the reader to YM for further examples.

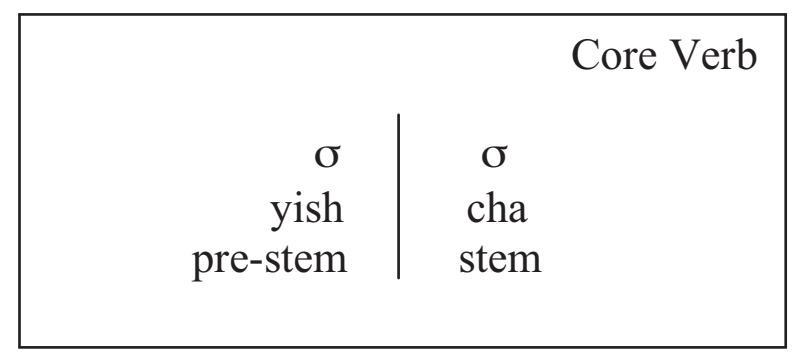

\footnotetext{
${ }^{1}$ For discussion of the structure of the Young and Morgan grammars and dictionaries, see the final chapter in McDonough (2003).
} 
We call attention to the two components, the pre-stem and stem units as distinct units, the two basic parts that make up the meaning unit "word." These units may vary independently, though the combinations are apparently not always productive. One overriding question concerns the speaker's tacit knowledge of this structure. Can we demonstrate that these units do vary independently? How productive is the system? Do the often rich specifications of the pre-stem limit the interpretation of the stem? One piece of evidence that they do comes from the regularities in the lexicon itself.

Consider the verb stem, the final syllable in the verb word. The verb stems in Athabaskan are "classificatory" stems, that is they refer to specific properties of objects or movement, for instance, such as the way an object is handled, its plurality or its physical shape. An example of this is the verbs of eating in Navajo. How eating is spoken about depends on what is being eaten; this specification is in turn encoded by the verb stem:

$\begin{array}{llll}\text { 'I eat... } & \text { yish ghał } & \text { meat } & \text { 'rolling, turning manner' } \\ & \text { yish keed } & \text { donut } & \text { 'sliding manner' } \\ \text { yish chozh } & \text { cabbage } & \text { 'leafy' } \\ \text { yis ts'ééh } & \text { ice cream } & \text { 'mushy' }\end{array}$

The verb stem refers to the way things move: /ghat/ is the basis for verbs that refer to the basic motion of rolling in a turning manner, and also shows up in various constructions with meanings such as 'to look about' (roll the eyes), to describe water rushing out of a canyon, to lie around (YM 1987:g329). ${ }^{2}$ The stem /keed/ is not confined to eating; it refers to sliding in a slow, dragging manner, and also shows up in the verb that refers to a slide show. The stem /choosh/ refers to 'a flat, leafy object' and shows up in 'to graze', /ts'ééh/ refers to mushy matter, etc. Another way of looking at this is to consider these activities in Navajo (Young 2000); all of these are related by having the same verb stem /dzíiz/, translated as 'drag, pull':

$$
\begin{aligned}
& \text { Activities designed using the stem: } \\
& \text { - } d z i 1 \text { íz } \\
& \text { 'pull, drag, or tow' }
\end{aligned}
$$

pull bag off a truck haul a trailer pull a sheep out of a corral tow a log out of the road to midwife pull a car into a shop sideswipe something pull a splinter out drag $\log$ and add it to a pile

\footnotetext{
${ }^{2}$ YM's 1987 The Navajo Language is divided into two books with separate numbering. These are designated as 'g' (grammar) and 'd' (dictionary), as in 'd139' (= page 139 in the dictionary).
} 
Mary Ann Willie (p.c.) has proposed that a native fluent speaker, versus a nonfluent speaker, has access to this structure (and it is the case that Navajo word play is based on this kind of variation). ${ }^{3}$ We refer you to the stem and root dictionary in YM and Young (2000) for extensive discussion of the stems and the meanings they participate in.

To the left of the stem is the pre-stem domain. The pre-stem contains, as a base, the traditional grammatical elements that all core Athabaskan verbs have, which include tense/aspect and subject agreement marking (yish = øimperfective/ $1^{\text {st }}$ person singular, a synthesis of positions VII ('Mode') and VIII ('subject agreement') in the template). YM consider these to be fusional elements, and they list them as conjugational paradigms. Calling these elements the Base Paradigms (YM g:200-201), as the "base of all word formation," they list 16 distinct conjugation patterns. That is, the realization of this fusional morpheme is paradigmatic and not combinatorial. McDonough $(1990,2000,2003)$ has called these base morphemes the Aux stem; S. Rice (p.c.) calls them TAM/A (Tense/Aspect Marking with Agreement), a term we adopt. The pre-stem can also contain a very rich set of prefixal material from both the disjunct and conjunct domains, which may significantly alter the context a verb word may apply to. But overall, the base of the domain, sitting at the right edge, is the TAM/A conjugations. In the example below, the $1^{\text {st }}-3^{\text {rd }}$ person singular and dual of the $\varnothing$ imperfective conjugation is listed, with the verb stem /tchin/. This is a canonical conjugation and it is often used to represent the "underlying" subject agreement morpheme set in templatic analyses. Again we refer the reader to YM for the differences between the 16 conjugations and their combinatorial possibilities.

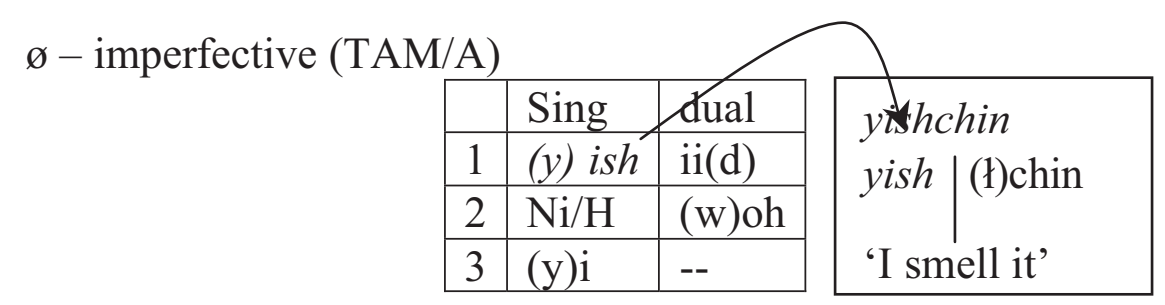

To the immediate left of TAM/A may fall a set of morphemes that alter the aspectual nature of the event signified by the word (position VI morphemes in the YM template). These are a difficult set of morphemes to define, both in terms of their morphophonemics and their semantics. In describing them YM (1987:g80) state:

Elements that no doubt were distinct in form and transparent in meaning historically have converged in shape... For some the meaning remains sufficiently transparent to permit definition, while for others meaning is obscure and definition is speculative or impossible on the basis of available data.

\footnotetext{
${ }^{3}$ See also Supalla's (1987) work on ASL classificatory verbs.
} 
This is a very rich and problematic area. YM refer to many of these as "subaspectual" morphemes, as they contain marking for "subaspects" to the conjugational paradigms (basically: imperfective, perfective, optative, and future), such as the seriative, inceptive, iterative, terminative, repetitive, revisionary, inchoative. A full discussion of these interesting morphemes is beyond the scope of this paper and we refer the reader to the extensive and excellent discussion in YM. What is important for us the concatenative relationship between the pre-stem entity and the verb stem.

To see the nature of this problem, note the following example. The stem is the same, /baaz/, meaning 'a hooplike or circular object' as it is used in the verb 'to drive a wheeled vehicle'. The pre-stem carries the information about how the stem / baaz/ and its referent 'a hooplike object' 4 are involved in the verbal activity stipulated by the verb word:

\begin{tabular}{|c|c|c|c|}
\hline \multicolumn{4}{|c|}{ pre-stem | stem (łbạ́ąz) } \\
\hline ni'sé & łbąaąz & 'to drive to a des & on and return' \\
\hline nánís & bááz & 'to return in it' & \\
\hline há'dis & bạaąz & 'to go after it' & \\
\hline na'as & bạáz & 'to drive around' & (YM d871) \\
\hline
\end{tabular}

The pre-stem domain combines with the stem to form these various meanings. In the present discussion, these subaspectuals are the morpheme types we are interested in for the salience of their distinctions (to return in a car, versus drive around in a car). We propose that because these morphemes condition the way in which an object moves or gets handled, speakers are likely to be able to manipulate them independently of the stem.

The question we wanted to ask is: Is there a way that we can get at a speaker's tacit knowledge of these two separate parts of the verb without explicitly asking them for their judgments?

\section{Videos}

In this section, we describe initial results of a new methodology we are developing with the aim of further investigating speaker knowledge of verb structure. The technique we used was to make and present videos of an activity to speakers and ask them to describe the activity. To get at subaspects, we decided to see if we could induce speakers to produce constructions like the seriative. We chose to attempt to embody the seriative because it is a rather salient distinction in the way an object, specified by the verb stem, is handled or moves.

\footnotetext{
${ }^{4}$ It is by convention that 'a hooplike object' is used in constructions that refer to driving a car, and we expect this type reference to vary across the language communities.
} 
Seriative Subaspect "...performing the verbal sequence one after the other..." "...describes the verbal action as segmented in form..." YM 1987:166

For example, in the following examples are two forms of the verb with the stem /jaad/ 'handle plural objects', and an example of verb 'to go in plural objects'; both include the seriative lexeme $/ \mathrm{hi}-\mathrm{I}^{5}$ All the forms indicate plural objects, but the manner in which they are carried is different depending on the pre-stem domain.

hi- seriative 'subaspect' jáád 'handle plural objects'

$\begin{array}{rll}\text { nish jááh } & \text { 'I arrive carrying them' } \\ \text { yah 'ahish jááh } & \text { 'I carried them in one after another' } \quad \text { YM:1987:166 }\end{array}$

yah 'ahi kááh kááh 'go (+3)'

At issue was the question as to whether we could induce speakers to produce alternations in the pre-stem domain such as the seriative over a non-seriative construction. The logic behind this goal was based on the postulation that if our hypotheses about the underlying meaning and uses of the pre-stems were correct, we should be able to apply this knowledge to reliably create situations that would generate alternate responses. We wanted to see if we could induce speakers to produce variation in subaspect in the pre-stem domain, while maintaining the same verb stem, by showing them similar activities performed in different manners.

To accomplish this we made series of iMovie videos, using a video camera plugged into a Mac. This proved to be a quick and easy process to which native speakers responded quite well, thus excellent for fieldwork. The videos were of two types: ones we made informally at the University of Rochester varying an activity that we believed could be representative of a subaspectual difference (the seriative). This included a video of a popcorn eating activity (plural object, plural behavior), and included variations such as one person eating out of a bowl of popcorn, three people together eating out of the bowl, three separately walking up to the bowl and eating out of it. We predicted that the pre-stem domain, and crucially not the stem, would vary according to the changes in the type of behavior. The second type of videos were ones we made on site in the field, at the NLA summer Institute, while a group of people were in the kitchen preparing dinner: a food preparation video. In this, we videoed people making chicken enchiladas, in particular, the activity of tearing up cooked chicken: one man

\footnotetext{
${ }^{5}$ Recall that these elements tend to be synthetic and difficult to segment. The seriative has several allophones, including a vowel length distinction. See YM 1987:g86.
} 
pulling a chicken apart, and two, then three people tearing up the chicken with their hands.

Both types of videos were played in an informal way to native Navajo speakers, who were asked to describe the scene in the video. We were looking to see how speakers used the pre-stem and stem domains to describe the activity. The results indicate that speakers do manipulate the two parts of the verb in describing the types of actions in structured ways, but their ability to do so is very complex. In the following section we discuss two relevant examples of the data we obtained.

\section{Data}

The first video was one of people eating popcorn, made at the University of Rochester. The videos were informal, which turned out to be very beneficial, as speakers responded to their naturalness very positively. Our aim with this video was to elicit the seriative construction. That is, we hoped that speakers would agree on a single verb stem that would represent the activity (popcorn eating) and vary the pre-stem in describing the various ways the action was being depicted in the videos. The videos presented one person eating popcorn out of a bowl (a), three people eating out of a bowl (b), and three people going up to the bowl one at a time (c). We asked speakers to describe the actions and to write down their answers.

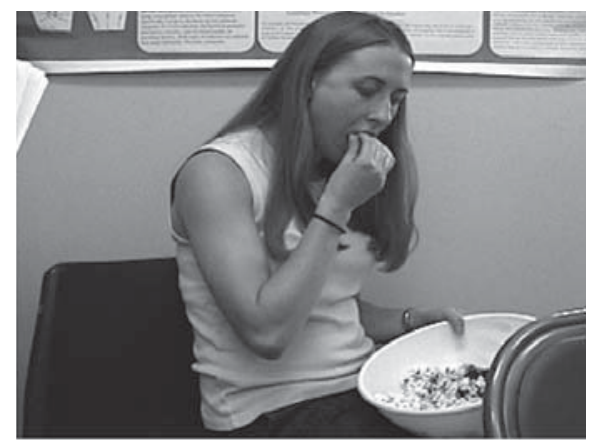

(a)

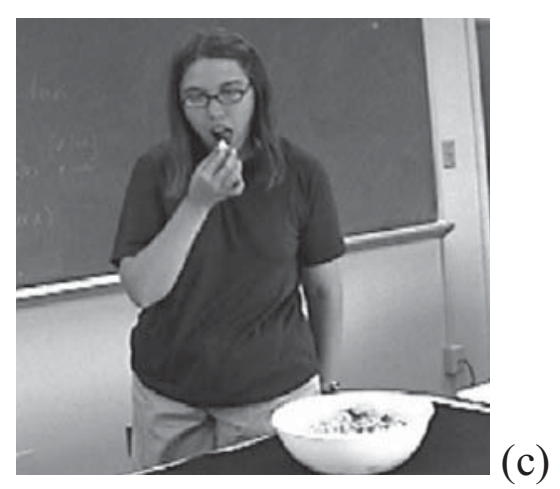

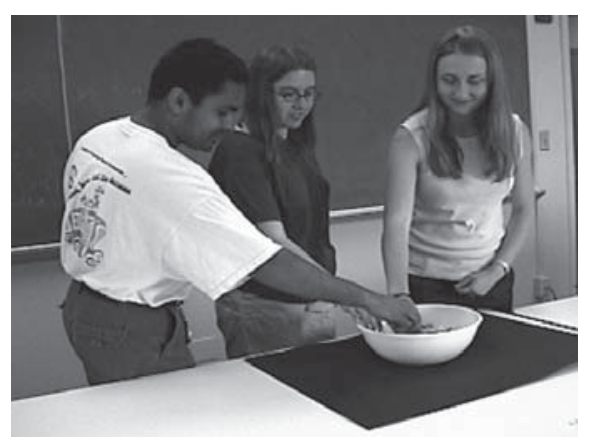

(b)

Examples of some of the constructions we got were as follows: 


$$
\begin{array}{r}
\text { aza 'yii jáá } \\
\text { aza yo t'áa } \\
\text { ayii jáá }
\end{array}
$$

Two of the three constructions used the disjunct postposition /aza-/ 'into the mouth' (YM 1987:d139). Speakers also used two different verb stems that referred to a salient distinction in the video which pertained to the manner in which the action took place: one actor (c), as opposed to the others, picked up popcorn one piece at a time; it looked quite polite in comparison. The others in the video were unceremoniously scooping up handfuls of popcorn and putting them into their mouths (a) and (b). The two verb stems are as follows:

$$
\begin{aligned}
& \text { jáá 'move, handle many small objects' } \\
& \text { t’ạ́ 'eat a hard object' }
\end{aligned}
$$

The action of the actor in (c) was described using the $/ t$ 'ál stem, 'eat a hard object'.

The pre-stem domain also varied. Two constructions which used a plural verb stem /jáál also contained the pre-stem morpheme /yii/, arguably containing subaspectual information (YM 1987:g83). The third, using the stem /t'ál, also contained subaspectual marking (YM:g85), though distinct from the ones appearing with /jáál.

A second example follows. This video was made in the kitchen of a dormitory that housed the participants and teachers of the Navajo Language Institute, summer 2003. Several people were preparing dinner consisting in part of chicken enchiladas. Preparing the chicken involved sitting at a table and tearing up a single cooked chicken (crucially, it turned out) with the fingers. We videoed one person doing the activity (a), two (b), and then three people working together to tear up the chicken. We were then able to play the video to native Navajo speakers who were in the room and ask them to describe the activity. We were able to effectively capture an activity on video and play it back while it was still within its context, thus presenting speakers with a cohesive and contained section of a shared activity to replay and discuss, while the participants still shared the activity. Given the richness of the specification within the verb and how little we understand it, providing a shared context is non-trivial. As we will see below, we got encouraging results.

As before, the speakers were asked to write down the words and phrases they used in describing the activity, though in the future we suggest recording these sessions using high quality audio recording techniques.

The action of this video involved tearing apart a cooked chicken with the fingers. As opposed to the previous video, in which one of the actors could be identified as doing something in a different manner, in this video, the activity 
across the actors was identical, and presented to speakers while the larger activity (meal preparation) was still occurring. Only the number of actors changed. (There was no difference between two and three actors.) We received a number of different constructions; for the most part they differed in the pre-stem domain rather than in the verb stem. Some examples of the elicited verbs are listed below, followed by a discussion. The forms in the orthographic representations were those given by the speakers; there are differences in tonal specification from that found in YM.

For the most part, for the verb stems, the speakers used forms of the verb stem /dlaad/, which participates in verbs meaning 'to tear or pull', the most salient aspect of the activity.
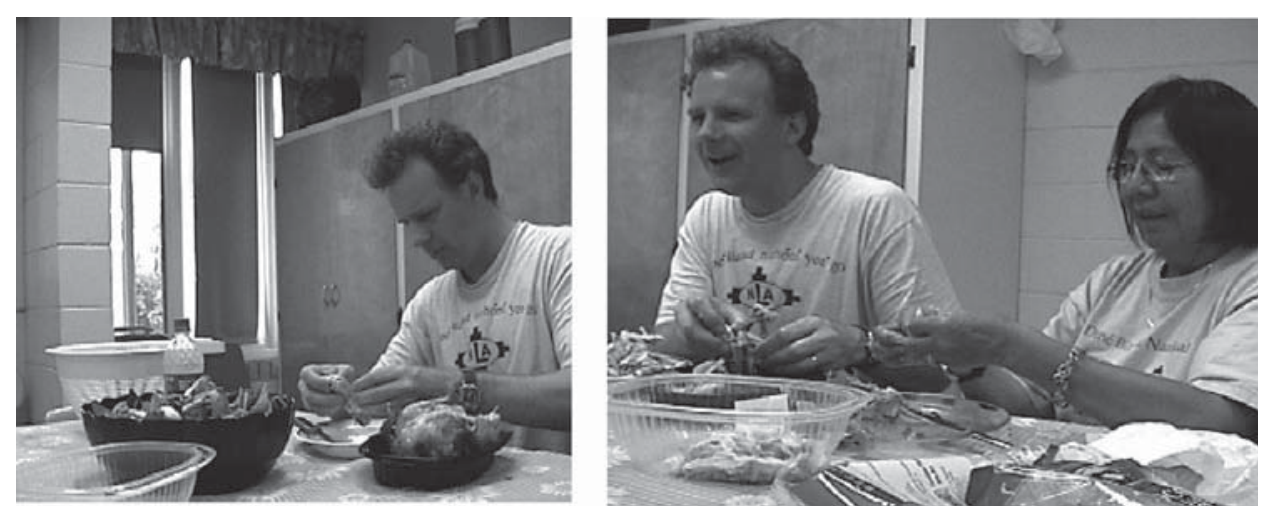

\section{niyi łdlah}

niyiis dlaad

nayii ldlaad

niyií nish

niyi lts'

\section{ahanis dlaad \\ ahandini tdlaad}

Speakers also used two other verb stems: most common was /nish/, a generic stem that is used in constructions referring to work, but to which YM also assign the meaning 'tear'. Another form used was the stem / tts ' $i$ '/, which refers to a pinching movement made with the fingers.

However, the greatest differences were in the pre-stem domain. For this discussion we separate these into two groups (as above): the $/ n /$ initial and the /aha/ initial, and discuss them briefly. The $n$-initial fall into two groups: the /ni/ 'terminative, cessative' and the /nal 'around about' (YM 1987:g37 and references therein); both are disjunct prefixes. Within the conjunct domain several exhibit the long vowel indicative of some type of subaspectual variation. Only one of 
them, /niyitdlah/, appears to have a simple conjunct construction made up of a single TAM/A element (nperf/3rd):

$$
\begin{array}{ll}
\text { niyitdlah } & \\
\text { ni } & \text { \# yi } \\
\text { 'term, cessative' \# nperf/3rd } & \text { ['tear, pull'] }
\end{array}
$$

Thus we find speakers responding to an identical activity by using distinct prestem combinations. They were present at the activity depicted in the video, and it was presented to them as a video while the context in which they experienced it still held (in the kitchen cooking).

Potential glosses for the lahal constructions are as follows (the disjunct morphemes are marked off by '\#' according to Athabaskan conventions; all glosses are from YM):

$$
\begin{array}{ll}
\text { ahanis dlaad } & \\
\text { [aha \# nis ] } & {[\text { 1-dlaad ] }} \\
\text { ['together' \# n-imper/1s] } & {[\text { trans- 'tear, pull'] }}
\end{array}
$$

ahandini łdlaad

[ahan \# di - ni ] [ ldlaad ] (YM 1987:d39) ['together' \# 'act with hands' - n-imper/1s] [ trans- 'tear, pull' ]

Apart from agreement marking $\left(1^{\text {st }}\right.$ versus $\left.3^{\text {rd }}\right)$ the differences between these two constructions are solely in the presence of the / di-/ 'hands or arms' in one of them. They differ from the previous examples in the shape of the pre-stem domain.

In several instances we asked speakers if they could comment on the constructions they chose. One speaker, when asked about her construction Iniyiinish/, replied that the /niyili/ part (our pre-stem) was "the pinching part" of the meaning. ${ }^{6}$ To us this potentially signifies two testable things: that speakers extract some understanding of the meanings of the pre-stem domain as independent units, thus they are at least partially combinatorial, and that the pre-stem domain may well condition the interpretation and choice of the stem. Also, the manner in which an activity is performed is highly relevant to the chosen lexical item in two distinct ways, (1) in the choice of the make-up of the pre-stem complex and (2) in the choice of the verb stem.

We also found that the informality of the videos was a considerable benefit to the discussion of the activity, and we recommend that future work be done using native actors in natural situations.

\footnotetext{
${ }^{6}$ In a similar case, a native speaker deconstructed the word /názhnoodahil 'circle dance' (a type of dance). Knowing the stem /dah/ referred to 'dance or celebrate', she separated out the pre-stem, which contains the Inál 'encircling', to mean 'circle around'. She could not identify the aspect or agreement markers in the construction, though she could manipulate them.
} 
In summary, native Navajo speakers exhibit behavior that indicates they possess independent access to both parts of the verb. As to the question of how important is the aspect of the pre-stem to expressing events, the answer was confounded by many things, likely to include exposure to the variations of the pre-stem domain. This is a testable hypothesis.

\section{Conclusion}

In conclusion, using the videos in the field to work with native speakers has proved to be a viable technique. The small videos provided focus for discussion of verb forms among native speakers. It provided them with a context for discussing different verb forms and vocabulary items. Several speakers sat around the computer and discussed the videos, attention was focused on the videos, and the conversation and discussion were lively. This activity allowed us to record the session in which speakers were using language in a unselfconscious way. Thus this also provided a potential source for corpus work, including recorded dialogue. The technique gets at knowledge of structure: what speakers choose to vary can tell us something about what they have access to and how productive the morphemes are.

To do further work, we need to work with native speakers. Working with a parsimonious model of the internal structure of the verb, we need to develop more controlled experimental materials, including especially videos, that are developed in conjunction with native speakers, and that reflect patterns in the lexicon. The body of data that comes out of recorded sessions, of both the video recordings and the discussion of them, is likely to yield a rich set of material to analyses that will help us understand the nature of the verb and its combinatorial base. Finally, because of the similarity of the languages within the family, this video technique and the materials developed for one Athabaskan language are likely to be useful across the Athabaskan family.

\section{References}

McDonough, J. 1990. Topics in the Morphology and Phonology of Navajo Verbs. Ph.D. diss., University of Massachusetts at Amherst.

McDonough, J. 2000. On the bipartite model of the Athabaskan verb. In T. B. Fernald and P. R. Platero, eds., The Athabaskan Languages: Perspectives on a Native American Language Family, 139-166. Oxford: Oxford University Press.

McDonough, J. M. 2003. The Navajo Sound System. Dordrecht: Kluwer.

Young, R. W. 2000. The Navajo Verb System: An Overview. Albuquerque: University of New Mexico Press.

Young, R., and W. Morgan. 1987. The Navajo Language. Albuquerque: University of New Mexico Press. 
Departments of Linguistics and Brain and Cognitive Sciences

503 Lattimore Hall

University of Rochester

P.O. Box 270096

Rochester, NY 14627-0096

jmmcd@ling.rochester.edu

rss@ling.rochester.edu 